\title{
Carnets
}

Revue électronique d'études françaises de l'APEF

Première Série - 2 Numéro Spécial 10-11 | 2011

D'un Nobel l'autre

\section{Le promontoire de la langue : variations sur une métaphore dans trois petits traités de Pascal Quignard}

\section{Cristina Álvares}

\section{OpenEdition}

Journals

Édition électronique

URL : http://journals.openedition.org/carnets/5337

DOI : 10.4000/carnets.5337

ISSN : 1646-7698

Éditeur

APEF

Édition imprimée

Date de publication : 1 janvier 2011

Pagination : 9-17

Référence électronique

Cristina Álvares, «Le promontoire de la langue : variations sur une métaphore dans trois petits traités de Pascal Quignard », Carnets [En ligne], Première Série - 2 Numéro Spécial 10-11 | 2011, mis en ligne le 16 juin 2018, consulté le 01 mai 2019. URL : http://journals.openedition.org/carnets/5337 ; DOI : $10.4000 /$ carnets.5337

\section{(2) $(1) \Theta$}

Carnets est mis à disposition selon les termes de la licence Creative Commons - Atribution - Pas d'utilisation commerciale 4.0 International. 


\title{
LE PROMONTOIRE DE LA LANGUE \\ Variations sur une métaphore dans trois petits traités de Pascal Quignard
}

\author{
Cristina Álvares \\ Universidade do Minho \\ calvares@ilch.uminho.pt
}

\begin{abstract}
Résumé
Dans ses petits traités, Pascal Quignard réfléchit sur le langage et ses rapports avec le réel, le sexe et la nature. J'analyserai la transformation substantialiste de la perception du langage à travers la métaphore du bout de la langue dans les traités publiés en 1993 (Le nom sur le bout de la langue), 1994 (Le sexe et l'effroi) et 1995 (Rhétorique spéculative): de forme à substance, de défaillance à maîtrise, de logos à physis. Dans cette transformation, qui implique aussi l'écriture, Le sexe et l'effroi (1994), centré sur l'ancien concept de phallos, joue un rôle médiateur.
\end{abstract}

\begin{abstract}
In his petits traités, Pascal Quignard reflects on language in connection with the real, sex and nature. The topic I will address is the substantialist transformation of the perception of language through the metaphor of the tip of the tongue in the treatises published from 1993 (Le nom sur le bout de la langue) through 1995 (Rhétorique Spéculative): from form to substance, fainting to mastering, logos to physis. In this transformation, which also concerns the literary writing, Le sexe et l'effroi (1994), stressing the Ancient concept of phallos, is given a prominent role.
\end{abstract}

Mots-clés: Métaphore, substance, écriture, phallique

Keywords: Metaphor, substance, writing, phallic 


\section{Postmoderne et substance}

L'Origine - irrémédiablement perdue mais dont il reste un petit quelque chose, appelé 'sordide' - est le référent majeur des fictions et des réflexions quignardiennes. L'Origine est la désignation de l'Être - puisque c'est là, à la source, c'est alors, Jadis, que l'on a vraiment existé, que l'on a réellement été, et c'est pourquoi la perte de l'Origine a fait de nous des 'ombres errantes', des être de désir. L'origine est appelée aussi 'le fauve' et 'le nu': le langage nu (lettre), la musique nue (chant des sirènes), la nudité des dieux (phallus); mais aussi la violence prédatrice. L'approche quignardienne de l'Origine semble osciller entre vide (le blanc, la nuit, l'absence, la scène invisible) et plein: présence massive et substantielle de quelque chose de vivant, le comble du vivant. Pour faire contraste modernité et postmodernité, Zizek les représente par un écrivain: Beckett serait l'écrivain moderne car chez lui la réalité (discours, activités, liens) s'ordonne autour d'un trou, l'absence de Godot nom du référent qui n'existe pas; de son côté, Kafka serait l'écrivain postmoderne puisque dans ses fictions la place centrale du référent, loin d'être vide, est bel et bien remplie par la présence écrasante de quelque chose de féroce et d'obscène (Zizek,1997:145-6). En transposant à Quignard, on pourrait dire que le traitement qu'il fait de l'Origine vacille entre Beckett et Kafka et que ces deux tendances ne sont pas mutuellement exclusives, elles coexistent et se superposent même, avec des accents différents, à travers un trait commun qui est celui de la violence, la violence du réel hyperesthésique. Mon objectif est de montrer que les trois petits traités de 1993, 1994 et 1995 s'alignent selon une progression qui va de Beckett à Kafka: l'accent est mis d'abord sur le vide, le manque, le négatif (Nom sur le bout de la langue), puis sur la substance, la force vitale, la nature comme physis, c'est-à-dire un corps animée (on voit ici que le postmoderne - c'est un de ses traits - a le goût de l'ancien et même de l'archaïque), en passant par un stade intermédiaire représenté par Le sexe et l'effroi. Cette transformation substantialiste mobilise le rapport du langage à la nature: perçus comme ontologiquement étrangers l'un à l'autre dans Nom sur le bout de la langue et $L e$ sexe et l'effroi (langage comme forme et nature comme force), langage et nature ont la même ontologie dans Rhétorique spéculative (force).

\section{À la périphérie}

J'ai parlé de place centrale vide ou pleine. Mais il faut préciser que Quignard ne conçoit pas l'Origine comme étant au centre (de l'univers humain, du symbolique). Au contraire, elle se trouve à la périphérie, à l'extrémité, au bout. Remarquons que cette décentration du référent majeur prend tout son sens dans le cadre du projet littéraire de Quignard qui consiste à réhabiliter des textes que les grands discours catégoriels, 
génériques, canoniques, doxaux ont repoussé à la frange du monde, à la marge du groupe social, faute de les avoir enregistrés comme valeurs dans le grand texte de la mémoire collective. L'argument le plus efficace à mon avis pour procéder à cette 'périphérisation' de l'Origine consiste dans la réappropriation, dans Rhétorique spéculative, de la thèse de Serge Moscovici sur l'hominisation comme 'cynégétisation'. Selon lui, chez les primates, le mâle dominant, qui occupe le centre du territoire du groupe avec les femelles et les petits, repousse les mâles subadultes vers les marges pour les empêcher d'accéder aux femelles. On reconnaît le scénario darwinien à partir duquel Freud a composé sa fiction du parricide primordial. Pourtant Moscovici, et Quignard avec lui, racontent une histoire différente, dont le sens est centrifuge. À la périphérie, les jeunes mâles s'exposent aux attaques des prédateurs. La solution n'a pas été celle, racontée par Freud, de la horde qui vient vers le noyau central pour y tuer le père. Sidérés et fascinés par la férocité des fauves, les mâles subadultes se sont mis à les imiter. C'est le début de leur 'cynégétisation': mimer les prédateurs, faire comme eux, les élever à la dignité de modèle, les manger (Quignard, 1995:37-40). Ce scénario archaïque permet à Quignard de s'éloigner du mythe freudien, 'mythe d'homme', et de le réécrire dans le sens centrifuge de Moscovici: la violence originaire n'est pas humaine (familiale, parentale), elle est animale et a eu lieu à la limite du territoire, à la frontière.

On comprend ainsi la fréquence dans le texte quignardien des lieux de bord, depuis le jardin de Mascarenhas dans Frontière jusqu'aux lieux sacrificiels que sont les promontoires et les falaises, en passant par la métaphore du bout de la langue: lingua, en latin, signifie la pointe du promontoire qui surplombe la mer (cf.1996:33). Lieu d'immolation, le promontoire signale la périphérie de l'ordre humain, ce bord où il touche à ce qui n'est ni ordre ni humain. Un individu est poussé jusqu'au bout du territoire ou de la langue, de la langue comme territoire, habitat du groupe, et se tient face à l'abîme: "C'est ainsi que la lingua est une Roche Tarpéienne et le flux des mots d'une foule poussant un homme qui tombe dans le vide vertical qui le sépare de la mer." (Quignard, 1996:35). Dans Boutès, Boutès, le seul des compagnons d'Ulysse qui ne s'est pas bouché les oreilles, se jette dans la mer, en répétant le saut du Plongeur de Paestum ${ }^{1}$ (dont la première occurrence se trouve dans Le sexe et l'effroi, p.228-30): "Les sociétés s'associent en poussant un homme d'un promontoire, hurlant un grand cri unanime qui apaise et qu'ensuite elles découpent sous forme de langage articulé" (Quignard, 2008:49).

\footnotetext{
${ }^{1}$ II y a dans le traitement quignardien du plongeon de Paestum une oscillation entre suicide et meurtre: est-ce un sacrifice par lequel le groupe se restaure ou est-ce le choix d'un dissident pour rejoindre la source, la mer/mère (cf.2008:75)? Ou les deux? Quignard affirme que la mort de Boutès est la scène symétrique à la lapidation et démembrement d'Orphée par les bacchantes (2008:69). Mais il est aussi celui qui saute hors du groupe et hors du langage (idem:19,33).
} 
Abyssos en grec signifie une bouche ouverte sans fond (1995:129), ce qui évoque la gueule du fauve et l'épouvante de la prédation originaire. Aussi Quignard écrit-il que "le bondissement des fauves est à la source du plongeur" (Quignard, 2008:51). Le nom sur le bout de la langue, oublié, est le point, ou la pointe, où la langue reflue et fait défaut. Déserté par le langage, l'être non parlant se tient, comme la victime sacrificielle, au-dessus de l'abîme. Il se tient sur le promontoire de la langue.

Ma mère se tenait toujours à l'extrémité de la table à manger, le dos à la porte de la cuisine. Brusquement, ma mère nous faisait taire. Son visage se dressait. Son regard s'éloignait de nous, se perdait dans le vague. Sa main s'avançait au-dessus de nous dans le silence. Maman cherchait un mot. Tout s'arrêtait soudain. Plus rien n'existait soudain.

Éperdue, lointaine, elle essayait, l'œil fixé sur rien, étincelant, de faire venir à elle dans le silence le mot qu'elle avait sur le bout de la langue. (Quignard, 1993:55-6).

\section{Phallicisation du corps et du langage}

Je voudrais maintenant attirer l'attention sur la dimension phallique du bout ou promontoire de la langue, ainsi que des figures de saillance qui lui sont associées. Dans $L e$ Nom sur le bout de la langue, le corps pétrifié de celui qui a perdu le nom se phallicise: "Cette tête qui se dresse soudain, la contemplation du corps qui cherche à faire revenir le mot perdu, ce regard parti au loin, ce regard impliqué dans la recherche de ce qui ne peut revenir - l'ensemble de cette tête est impérieusement sexuel" (Quignard, 1993:70). Ajoutons les statues phalliques du jardin de Frontière qui pointent le vide:

Il y avait une Diane de marbre blanc qui visait avec la lance l'air, le ciel du soir, le néant. Ou encore un grand Cupidon ailé qui visait avec sa flèche le vide de la toile. Un dieu Orphée qui jouait de la viole et poussait du bout de l'archet le néant. Un Brutus qui pointait avec sa petite dague le néant. Un grand Priape de marbre qui plongeait l'extrémité de son sexe vigoureux dans le néant et l'air et le silence. (Quignard, 1995:37)

Ces statues sont des déïctiques. Elles indiquent le référent majeur, ce que vise le désir nostalgique: l'avant-monde, l'Origine. De même, le corps pétrifié pointe le trou hyperesthésique que "le réel d'avant le langage ${ }^{2}$, le réel d'avant la réalité", creuse au bout du monde (Quignard, 1993:76).

\footnotetext{
${ }^{2}$ Bien qu'il s'inspire du réel lacanien, surtout pour ce qui est de son antinomie au symbolique, au savoir et à la réalité, le réel quignardien est différent de par sa qualité originelle, animale et abyssale. Le réel quignardien est naturel, si bien qu'il se confond avec la mort biologique.
} 
Mais au lieu du désir nostalgique et en quelque sorte contemplatif (comme celui de Perceval pensif abandonné au vide), l'érection du corps signale aussi le désir au comble de l'angoisse ${ }^{3}$. Dans Le sexe et l'effroi, Quignard parle de l'immobilité du fauve juste avant de bondir sur la proie, ainsi que de la sidération de la proie face à la gueule béante du fauve ou encore de la stupeur qui frappe ceux que la vision du sexe aveugle. C'est l'instant de mort, qui est "le plus vivant du vif sur le vif" (de même que l'érection est pétrification): la tension maximale du corps, sa suspension au dessus de l'abîme, sa saillance pétrifiée prolongeant celle du promontoire dans l'instant même qui précède le saut du plongeur à pic, son élan, son jet, sa mort. L'élan de la vie est l'élan de la (mise à) mort. C'est le même jet. Jactance/jactura signifie sacrifice (Quignard, 1995:129, 2008:50) et de même que la stupor de l'instant de mort précède et prépare le bond du fauve ou le vol piqué du rapace, l'érection débouche sur l'éjaculation: retrouver le mot perdu sur le bout de la langue, c'est éjaculer (cf.Quignard, 1993:72, 73).

Finalement, dans Rhétorique spéculative, c'est le langage lui-même qui se phallicise au moyen de la lettre. La littérature, "souci atomique des lettres", est la nudité du langage qui saillit, "ce qui tend et se tend comme lors du désir masculin" (Quignard, 1995:63-4). Les figures de rhétorique sont "des saillies qui font brèche, qui déchirent le langage lui-même", qui le mettent à nu (Quignard, 1995:72). La littérature est le langage conçu comme arme de jet, jactance. Le logos, c'est-à-dire le langage nu, est à pic au-dessus du gouffre: "L'à-pic est ce qui s'ouvre sous l'humain comme abîme, comme la falaise tombe à pic. L'humain fuit l'abîme. Le logos seul l'y ramène" (Quignard, 1995:73).

\section{Du langage-logos au langage-physis}

Cette sorte de continuité entre corps et langage (phallicisés) que l'on dégage du motif du promontoire de la langue ne doit pas nous cacher que Le nom sur le bout de la langue et Rhétorique spéculative sont deux traités qui présentent chacun une perspective très différente sur le langage. Rhétorique spéculative énonce explicitement ce renversement de perspective. Dans Nom sur le bout de la langue nous trouvons une valorisation de la défaillance du langage en tant que condition de l'écriture: "[L'écrivain] se consacre (...) au livre ouvert comme la bouche est ouverte sur le mot défaillant qu'elle sur le point de recouvrer, qu'elle va ressusciter plus vivant que si elle l'avait su“ (Quignard, 1993:100). Par contre, Rhétorique spéculative méprise une telle défaillance: "Il est hors de question que l'empereur de Rome puisse se trouver dans le cas de figure d'un homme en train de

\footnotetext{
${ }^{3}$ Désir et angoisse ne sont pas discernables. Tout désir angoisse. Le désir nostalgique n'est pas exempt d'angoisse. L'angoisse de celui qui va sauter dans le gouffre n'est pas exempt de nostalgie: c'est le cas de Boutès.
} 
chercher, en plein Sénat, devant les Pères, un nom sur le bout de sa langue, bouche béante" (Quignard, 1995:30). Au langage défaillant, Rhétorique spéculative oppose le langage maîtrisé et attaque le postulat inaugural du Nom sur le bout de la langue selon lequel l'écrivain et l'enfant (in-fans) habitent le défaut du langage (Quignard, 1993:9-10): "L'écrivain est celui qui choisit son langage et n'en est pas dominé. II est le contraire de l'enfant" ${ }^{4}$ Quignard justifie la différence de perspectives dans le même petit traité: "C'est la grande nature, le fond de l'art. Et ce qui est défaillance aux yeux de l'homme ne l'est pas à ses yeux" (Quignard, 1995:73). II suggère que le Nom sur le bout de la langue exprime le point de vue de l'homme, alors que Rhétorique spéculative exprime celui de la nature. Jusqu'en 1995 Quignard avait souligné dans le langage sa dimension seconde de compétence acquise, voile fragile et défaillant sur la violence de la vie (Carus, Nom sur le bout de la langue). Cette perception du langage comme étant ontologiquement étranger à la nature culmine dans Nom sur le bout de la langue: c'est lorsque le langage fait défaut que l'homme se trouve au bord de sa condition première, de son être originaire:

Qu'un mot puisse être perdu, cela veut dire: la langue n'est pas nous-mêmes. Que la langue en nous est acquise, cela veut dire: nous pouvons connaître son abandon. Que nous puissions être sujets à son abandon, cela veut dire que le tout du langage peut refluer sur le bout de la langue. Cela veut dire que nous pouvons rejoindre l'étable ou la jungle ou l'avant-enfance ou la mort (Quignard, 1993:58). ${ }^{5}$

Dans ce cadre, le langage est un voile qui couvre et apaise la voracité de la vie (la gueule grande ouverte de l'abîme ou du fauve), une forme qui organise et stabilise le réel en monde, qui établit le "status quo ontologique" comme Ersatz. De 1979 (Carus) jusqu'à 1993 l'idée prévaut selon laquelle le retrait (reflux) du langage-voile est corrélatif du vide originaire (l'origine comme négatif) - 'le blanc qui est à notre source' (Quignard1993:70).

Mais Rhétorique spéculative enracine le langage dans la nature et le redéfinit comme force. II faut entendre 'nature' ici au sens héraclitien de physis: transformation torrentueuse (cf.1995:54-5). C'est la nature-physis qui est l'unique source et le fondement du logos (cf. 1995:62). L'origine n'est pas un négatif (le non-être) mais la positivité de la substance: "nous appartenons à la nature par le sperme, par la nuit, par la chair, par le sang, par la mort"

\footnotetext{
${ }^{4}$ Chantal Lapeyre-Desmaison a bien vu que Rhétorique spéculative marque un tournant en ce qui concerne le rapport de l'écriture au langage. La lettre étant dans le langage un excès interne, ce qui dans la structure même du langage le fait saillir, le pousse au-delà de sa propre loi et lien, littérature et langage ne sont plus dans un rapport 'relativement aporétique' (Lapeyre-Desmaison2006:72). En effet, Rhétorique spéculative ne conçoit pas le défaut du langage comme condition et fondement de la littérature. De là à penser que l'écriture n'est plus mise au silence du langage, c'est une autre question. D'ailleurs, ce n'est pas sûr que Quignard ne soit pas revenu, dans des textes postérieurs à Rhétorique spéculative, à la définition du langage comme voile.

${ }_{5}$ Quignard affirme dans l'entretien avec Chantal Lapeyre-Desmaison: "Notre âme est toute entière langue, mais nous ne sommes pas qu'âme. Nous ne sommes pas qu'occupation culturelle. De l'origine, de l'a-parlance, de l'abîme, du corporel, de l'animal, de l'insublimable persistent en nous" (2001:102)
} 
(Quignard, 1995:135). Dans ce cadre, le langage n'a plus rien d'une formation seconde, au contraire il est premier, puissant, violent. II n'est pas un logos prédécoupé, une structure ou forme à appliquer sur une substance, un ordre symbolique stabilisant. Bien au contraire, le langage est, grâce à la lettre, organe, substance, force vitale: "[...] mais au langage in germine, à la semence originaire, germinative, à la littera, à la substance littérale et pathique du langage, à la chose littéraire". (Quignard, 1995:31). Et plus loin: "Le littéraire est cette remontée de la convention à ce fonds biologique dont la lettre ne s'est jamais séparée" (Quignard, 1995:47). Cette conception naturaliste du langage se combine au pouvoir qu'a le rhéteur de le maîtriser. Son art consiste à intensifier dans le langage la violence de la nature pour faire apparaître l'instabilité de sa morphologie.

[...] dans le monde romain, la force du style est décrite comme un jet irrépressible, où le pathos prend pour icône le torrent [...]. Cette force est liée à la violence de la nature elle-même. [...] Les figures de rhétorique [sont] des accélérateurs de flux, des canalisations ou des dispositifs qui précipitent le cours du langage en torrent, qui l'exhaussent en montagne, ou qui le rompent en abîme. (Quignard,1995: 63, 65-6).

Percevoir le langage du point de vue de la nature, c'est saisir sa part de substance, de continu (flux, torrent), de violence, autrement dit ce qui du et dans le langage relève de la physis: son noyau asème, la lettre (l'être du langage). Rhétorique spéculative supprime la différence ontologique (l'écart) entre logos et pathos (Quignard, 1995:23), entre "la discontinuité du langage voué à la dissidence des objets et impliqué dans l'identification des individus - la face vue par miroir - et le continu maternel, le fleuve, le jet d'urine maternel la face vue en face" (Quignard, 1993:94).

Revenons à la connotation phallique de la métaphore du bout de la langue. Elle implique une conception pudique du langage comme voile, lequel, en défaillant, découvre un corps phallicisé à l'instant de mort, prolongeant dans sa pétrification le promontoire d'où il va plonger. Mais dans la conception physique (littéraire, rhétorique) du langage, c'est le langage même qui est nu et se présente sous les traits du phallos-fascinus du Le sexe et l'effroi: "le logos jaillit de la bouche comme le sperme à l'extremité du fascinus" ${ }^{2}$. Rhétorique spéculative a sans doute été préparée par le traité de l'année précédente Le sexe et l'effroi. Dans les deux traités il est question de l'Antiquité gréco-romaine: la sexualité (aussi bien privée que rituelle, sacrée) dans Le sexe et l'effroi; et la tradition antimétaphysique de la rhétorique spéculative avec Fronton, Marc Aurèle, le pseudo-Longin, dans Rhétorique spéculative.

\footnotetext{
${ }^{6}$ En sanscrit, linga signifie le signe et le sexe mâle: Shivalinga $(m)$ désigne le phallus du dieu hindu Shiva. Des spécialistes d'histoire des religions, comme M. Eliade, et de mythologie hindou ou comparée, comme G. Dumézil, l'ont affirmé. Voir aussi M. Coquet, Linga, Le signe de Shiva, Paris, Deux Océans, 2002.
} 
Le sexe et l'effroi a une position chronologiquement médiatrice entre Nom sur le bout de la langue et Rhétorique spéculative et il semble avoir joué un rôle dans le passage de la conception logique (langage-voile) à la conception physique du langage (langage-phallus). Je vais juste indiquer les lignes majeures du traité qui s'oriente de façon parfois hésitante vers un naturalisme substantialiste que Rhétorique spéculative exprime sans ambiguïté. Le sexe et l'effroi affirme que "Physis en grec signifie aussi bien la nature que le phallos" (Quignard, 1994:97) et que "le fascinus est la divinité des dieux dévêtue" (Quignard, 1994:29). Les corps des dieux étant cependant diaphanes, des simulacra, des pellicules d'atomes (cf. 1994:167-8), ce qui apparait lorsque le voile tombe est encore un voile. Cette nature qu'est le phallus serait-elle sans substance? En effet, le réel sexuel, scène primitive invisible, semble relever au moins en partie du réel négatif du Nom sur le bout de la langue et c'est pourquoi le phallus est défini comme organe, soma, physique, tout en gardant son statut de chose invisible, divine, méta-physique. Corrélativement la fonction logique et pudique du voile attribuée au langage est fortement soulignée: l'enfant, "qui n'a pas encore accédé au langage, n'a pas encore accédé au voile: il voit encore la nudité originaire"; alors que les adultes "voient toujours un fascinus déjà voilé par le langage qui les fait hommes" (Quignard,1994:145). Au lieu que le retrait ou reflux du langage dévoile un corps phallicisé, ici, la phallophanie désarticule le langage - tout comme le corps de la victime est démembré dans l'orgie rituelle: "Dionysos (...) est le dieu qui rompt le langage. II court-circuite toute sublimation. II refuse la médiatisation des conflits. II déchire tout vêtement sur la nudité originaire" (Quignard, 1994:327). Bref, l'effet déchirant de la phallophanie sur le langage est le même que celui que Rhétorique spéculative attribue à la lettre, la différence essentielle consistant dans le fait que la lettre est interne au langage et que, par conséquent, le déchirement est dans la nature du langage même, alors que le phallus lui est extérieur. II y a dans Le sexe et l'effroi le phallus-physis d'un côté et, de l'autre, le langage-logos. Par contre dans Rhétorique spéculative cet écart est annulé.

En conclusion, le schéma qui suit systématise les différences entre les trois petits traités concernant la perception du langage.

\begin{tabular}{|l|l|l|}
\hline \multicolumn{1}{|c|}{ NBL 1993 } & \multicolumn{1}{|c|}{ SE 1994 } & \multicolumn{1}{c|}{ RS 1995 } \\
\hline Langage f Nature & $\begin{array}{l}\text { Langage = Nature } \\
\text { Logos = Physis/Phallos }\end{array}$ & $\begin{array}{l}\text { Langage = Nature } \\
\text { Logos = Physis }\end{array}$ \\
\hline Réel négatif : vide & Réel sexuel : scène & Réel naturel: substance \\
\hline Langage=voile réel vide & $\begin{array}{l}\text { Langage=voile réel voile } \\
\text { (simulacra) }\end{array}$ & Langage = réel violence vie \\
\hline Langage défaillant & $\begin{array}{l}\text { Langage déchiré (par } \\
\text { phallos) }\end{array}$ & $\begin{array}{l}\text { Langage saillant } \\
\text { autodéchirant } \\
\text { (par lettre) } \\
\text { Flux, torrent }\end{array}$ \\
\hline $\begin{array}{l}\text { Défaillance du langage } \\
\text { fonde écriture }\end{array}$ & $\begin{array}{l}\text { peinture } \\
\text { se fonde de la scène } \\
\text { invisible }\end{array}$ & $\begin{array}{l}\text { Littérature est mâtrise du } \\
\text { langage }\end{array}$ \\
\hline $\begin{array}{l}\text { Corps phallicisé } \\
\text { (pétrifié) }\end{array}$ & $\begin{array}{l}\text { Phallos (érection, } \\
\text { éjaculation) }\end{array}$ & $\begin{array}{l}\text { Langage phallicisé (jet, élan } \\
\text { rhétorique) }\end{array}$ \\
\hline
\end{tabular}




\section{Bibliographie}

LAPEYRe-Desmaison, Chantal (2006). Mémoires de l'origine. Paris: Flohic.

Moscovicl, Serge (1972). La société contre nature: Union Générale des Éditions.

QuignaRd, Pascal (1990). Petits Traités I. Paris: Gallimard/Folio.

QUIGNARD, Pascal (1992). La frontière. Paris: Gallimard/Folio.

QUIGNARD, Pascal (1993). Le nom sur le bout de la langue. Paris: Gallimard/Folio.

QUIGNARD, Pascal (1994). Le sexe et l'effroi. Paris: Gallimard/Folio.

QUIGNARD, Pascal (1995). Rhétorique Spéculative. Paris: Calmann-Levy.

QUIGNARD, Pascal (1996). La haine de la musique. Paris: Calmann-Levy.

QUIGNARD, Pascal (2008). Boutès. Paris: Galilée.

ZIZEK, Slavoj (1997). Looking awry. An Introduction to Jacques Lacan through Popular Culture, Cambridge, MA \& London, England: MIT Press. 
